\title{
Sensitivity of a tonne-scale NEXT detector for neutrinoless double-beta decay searches
}

\section{Onext}

\section{The NEXT collaboration}

C. Adams, ${ }^{20}$ V. Álvarez, ${ }^{25}$ L. Arazi, ${ }^{6}$ I.J. Arnquist, ${ }^{23}$ C.D.R Azevedo, ${ }^{4}$ K. Bailey, ${ }^{20}$

F. Ballester, ${ }^{25}$ J.M. Benlloch-Rodríguez, ${ }^{17}$ F.I.G.M. Borges, ${ }^{14}$ N. Byrnes, ${ }^{3}$ S. Cárcel, ${ }^{22}$ J.V. Carrión, ${ }^{22}$ S. Cebrián, ${ }^{26}$ E. Church, ${ }^{23}$ C.A.N. Conde, ${ }^{14}$ T. Contreras, ${ }^{11}$ A.A. Denisenko, ${ }^{2}$ G. Díaz, ${ }^{24}$ J. Díaz, ${ }^{22}$ J. Escada, ${ }^{14}$ R. Esteve, ${ }^{25}$ R. Felkai, ${ }^{6,22}$ L.M.P. Fernandes, ${ }^{13}$ P. Ferrario, ${ }^{9,17}$ A.L. Ferreira, ${ }^{4}$ F. Foss, ${ }^{2}$ E.D.C. Freitas, ${ }^{13}$ Z. Freixa, ${ }^{9,18}$ J. Generowicz, ${ }^{17}$ A. Goldschmidt, ${ }^{8}$ J.J. Gómez-Cadenas, ${ }^{9,17, a}$ R. González, ${ }^{17}$ D. González-Díaz, ${ }^{24}$ S. Gosh, ${ }^{11}$ R. Guenette, ${ }^{11}$ R.M. Gutiérrez, ${ }^{10}$ J. Haefner, ${ }^{11}$ K. Hafidi, ${ }^{20}$ J. Hauptman, ${ }^{1}$ C.A.O. Henriques, ${ }^{13}$

J.A. Hernando Morata, ${ }^{24}$ P. Herrero, ${ }^{17}$ V. Herrero, ${ }^{25}$ J. Ho, ${ }^{11}$ Y. Ifergan, ${ }^{6,7}$ B.J.P. Jones, ${ }^{3}$ M. Kekic, ${ }^{24}$ L. Labarga, ${ }^{21}$ A. Laing, ${ }^{3}$ P. Lebrun, ${ }^{5}$ N. López-March, ${ }^{22}$ M. Losada, ${ }^{10}$ R.D.P. Mano, ${ }^{13}$ J. Martín-Albo, ${ }^{11,22}$ A. Martínez, ${ }^{22}$

M. Martínez-Vara, ${ }^{17,22}$ G. Martínez-Lema, ${ }^{6}$ A.D. McDonald, ${ }^{3}$ Z.E. Meziani, ${ }^{20}$ F. Monrabal, ${ }^{9,17}$ C.M.B. Monteiro, ${ }^{13}$ F.J. Mora, ${ }^{25}$ J. Muñoz Vidal, ${ }^{22}$ C. Newhouse, ${ }^{2}$ P. Novella, ${ }^{22}$ D.R. Nygren, ${ }^{3, a}$ E. Oblak, ${ }^{17}$ B. Palmeiro, ${ }^{22,24}$ A. Para, ${ }^{5}$ J. Pérez, ${ }^{12}$ M. Querol, ${ }^{23}$ A. Redwine, ${ }^{5}$ J. Renner, ${ }^{23}$ L. Ripoll, ${ }^{19}$ I. Rivilla, $,{ }^{9}, 17$ Y. Rodríguez García, ${ }^{10}$ J. Rodríguez, ${ }^{25}$ C. Rogero, ${ }^{16}$ L. Rogers, ${ }^{3}$ B. Romeo, ${ }^{12,17}$ C. Romo-Luque, ${ }^{22}$ F.P. Santos, ${ }^{14}$ J.M.F. dos Santos, ${ }^{13}$ A. Simón, ${ }^{6}$ M. Sorel, ${ }^{22}$ C. Stanford, ${ }^{11}$ J.M.R. Teixeira, ${ }^{13}$ P. Thapa ${ }^{2}$ J.F. Toledo, ${ }^{25}$ J. Torrent, ${ }^{17}$ A. Usón, ${ }^{22}$ J.F.C.A. Veloso, ${ }^{4}$ T.T. Vuong, ${ }^{2}$ R. Webb, ${ }^{15}$ R. Weiss-Babai, ${ }^{6, b}$ J.T. White, ${ }^{15, c}$ K. Woodruff ${ }^{3}$ and N. Yahlali ${ }^{22}$

\footnotetext{
${ }^{1}$ Department of Physics and Astronomy, Iowa State University, Ames, Iowa, U.S.A.

${ }^{2}$ Department of Chemistry and Biochemistry, University of Texas at Arlington, Arlington, Texas, U.S.A.

${ }^{3}$ Department of Physics, University of Texas at Arlington, Arlington, Texas, U.S.A.
}

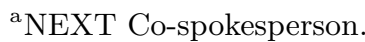

${ }^{\mathrm{b}}$ Now at Soreq Nuclear Research Center, Yavneh, Israel.

${ }^{\mathrm{c}}$ Deceased. 
${ }^{4}$ Institute of Nanostructures, Nanomodelling and Nanofabrication (i3N), Universidade de Aveiro, Aveiro, Portugal

${ }^{5}$ Fermi National Accelerator Laboratory, Batavia, Illinois, U.S.A.

${ }^{6}$ Unit of Nuclear Engineering, Faculty of Engineering Sciences,

Ben-Gurion University of the Negev, Beer-Sheva, Israel

${ }^{7}$ Nuclear Research Center Negev, Beer-Sheva, Israel

${ }^{8}$ Lawrence Berkeley National Laboratory, Berkeley, California, U.S.A.

${ }^{9}$ Ikerbasque (Basque Foundation for Science), Bilbao, Spain

${ }^{10}$ Centro de Investigación en Ciencias Básicas y Aplicadas, Universidad Antonio Nariño, Bogotá, Colombia

${ }^{11}$ Department of Physics, Harvard University, Cambridge, Massachusetts, U.S.A.

${ }^{12}$ Laboratorio Subterráneo de Canfranc, Canfranc-Estación, Spain

${ }^{13}$ LIBPhys, Universidade de Coimbra, Coimbra, Portugal

${ }^{14}$ LIP, Departamento de Física, Universidade de Coimbra, Coimbra, Portugal

${ }^{15}$ Department of Physics and Astronomy, Texas AछM University, College Station, Texas, U.S.A.

${ }^{16}$ Centro de Física de Materiales (CFM), CSIC $\&$ Universidad del País Vasco (UPV/EHU), Donostia-San Sebastián, Spain

${ }^{17}$ Donostia International Physics Center (DIPC), Donostia-San Sebastián, Spain

${ }^{18}$ Departmento de Química Orgánica I, Universidad del País Vasco (UPV/EHU), Donostia-San Sebastián, Spain

${ }^{19}$ Escola Politècnica Superior, Universitat de Girona, Girona, Spain

${ }^{20}$ Argonne National Laboratory, Lemont, Illinois, U.S.A.

${ }^{21}$ Departamento de Física Teórica, Universidad Autónoma de Madrid, Madrid, Spain

${ }^{22}$ Instituto de Física Corpuscular (IFIC), CSIC \& Universitat de València, Paterna, Spain

${ }^{23}$ Pacific Northwest National Laboratory, Richland, Washington, U.S.A.

${ }^{24}$ Instituto Gallego de Fúsica de Altas Energías, Universidade de Santiago de Compostela, Santiago de Compostela, Spain

${ }^{25}$ Instituto de Instrumentación para Imagen Molecular (I3M), CSIC \& Univ. Politècnica de València, Valencia, Spain

${ }^{26}$ Centro de Astropartículas y Física de Altas Energías (CAPA), Universidad de Zaragoza, Zaragoza, Spain

E-mail: next-src@pegaso.ific.uv.es

ABSTRACT: The Neutrino Experiment with a Xenon TPC (NEXT) searches for the neutrinoless double-beta $(0 \nu \beta \beta)$ decay of ${ }^{136} \mathrm{Xe}$ using high-pressure xenon gas TPCs with electroluminescent amplification. A scaled-up version of this technology with about 1 tonne of enriched xenon could reach in less than 5 years of operation a sensitivity to the half-life of $0 \nu \beta \beta$ decay better than $10^{27}$ years, improving the current limits by at least one order of magnitude. This prediction is based on a well-understood background model dominated by radiogenic sources. The detector concept presented here represents a first step on a compelling path towards sensitivity to the parameter space defined by the inverted ordering of neutrino masses, and beyond.

Keywords: Dark Matter and Double Beta Decay (experiments)

ARXIV EPRINT: 2005.06467 


\section{Contents}

1 Introduction 1

2 The NEXT experiment: concept and status 2

3 A NEXT detector with a tonne of xenon 4

$\begin{array}{lll}4 & \text { Backgrounds at the tonne scale } & 6\end{array}$

4.1 Natural radioactivity in detector materials 6

$\begin{array}{lll}4.2 & \text { Radon } & 9\end{array}$

$\begin{array}{lll}4.3 & \text { Backgrounds of cosmogenic origin } & 9\end{array}$

5 Signal efficiency and background rejection 10

6 Projected sensitivity to neutrinoless double-beta decay 15

$\begin{array}{llr}7 & \text { Discussion and conclusions } & 18\end{array}$

\section{Introduction}

Neutrinos are the only particles in the Standard Model that could be Majorana fermions, that is, completely neutral fermions that are their own antiparticles. Majorana neutrinos imply lepton number violation as well as the existence of new physics at an energy scale inversely proportional to the observed neutrino masses [1]. This new-physics scale provides a simple explanation for the striking lightness of neutrinos [2-5], and is possibly connected with the predominance of matter over antimatter in the universe [6].

The most sensitive known experimental method to verify whether neutrinos are Majorana particles is the search for neutrinoless double-beta $(0 \nu \beta \beta)$ decay [7-11]. In this hypothetical second-order weak process, a nucleus with atomic number $Z$ and mass number $A$ transforms into its isobar with atomic number $Z+2$ emitting two electrons only. The decay does not conserve lepton number $(\Delta L=2)$ and requires the neutrino be a Majorana particle.

No evidence of $0 \nu \beta \beta$ decay has been found so far. The best current limits on the halflife of the decay have been set by the KamLAND-Zen [12] and GERDA [13] experiments using, respectively, ${ }^{136} \mathrm{Xe}$ and ${ }^{76} \mathrm{Ge}$ as $0 \nu \beta \beta$ source:

$$
\begin{gathered}
T_{1 / 2}\left({ }^{136} \mathrm{Xe} \rightarrow{ }^{136} \mathrm{Ba}+2 e^{-}\right)>1.07 \times 10^{26} \text { years }(90 \% \mathrm{CL}), \\
T_{1 / 2}\left({ }^{76} \mathrm{Ge} \rightarrow{ }^{76} \mathrm{Se}+2 e^{-}\right)>1.80 \times 10^{26} \text { years }(90 \% \mathrm{CL}) .
\end{gathered}
$$

The experimental goal for the next generation of experiments is the exploration of the region of half-lives up to $10^{28}$ years. This will require exposures well beyond 1 tonne year 
and background rates lower than 1 count tonne $\mathrm{e}^{-1} \mathrm{yr}^{-1}$. Only a few of the experimental techniques presently considered will be able to attain those levels (see, e.g., [14, 15]).

In this paper we discuss the reach of a tonne-scale version of the Neutrino Experiment with a Xenon TPC (NEXT) considering only incremental improvements over the design of NEXT-100 [16, 17], the latest stage of the NEXT detector series, expected to start operation in 2022 at the Laboratorio Subterráneo de Canfranc (LSC), in Spain. Through a combination of good energy resolution, tracking-based event identification, radiopurity and shielding, a NEXT detector with active mass in the tonne range would be able to improve the current limits by more than an order of magnitude. The NEXT Collaboration is also pursuing a more disruptive approach to a tonne-scale experiment based on the efficient detection of the $\mathrm{Ba}^{++}$ion produced in the double-beta decay of ${ }^{136} \mathrm{Xe}$ using singlemolecule fluorescence imaging (SMFI) [18-22]. This technique has the potential to realize an effectively background-free experiment that could reach a sensitivity to the half-life of $0 \nu \beta \beta$ decay better than $10^{28}$ years, but it is still the subject of intense R\&D beyond the scope of the present article.

\section{The NEXT experiment: concept and status}

NEXT is an international effort dedicated to the search for $0 \nu \beta \beta$ decay in ${ }^{136} \mathrm{Xe}$ using high-pressure xenon gas time projection chambers (HPXeTPC) with amplification of the ionization signal by electroluminescence (EL). This detector technology takes advantage of the inherently low fluctuations in the production of ionization pairs (i.e., small Fano factor) in xenon gas to achieve an energy resolution significantly better than that of other ${ }^{136}$ Xe-based double-beta decay experiments [23]. Moreover, the tracks left in gaseous xenon by $0 \nu \beta \beta$ events have distinct features that can be used for background rejection.

Figure 1 illustrates the detection process in a NEXT HPXeTPC. The interaction of charged particles with the xenon gas is immediately followed by the emission of scintillation light, the so-called S1 signal. The ionization electrons left behind by the interacting particle drift under the influence of an electric field towards another region of the detector, the $E L$ gap, with an electric field of higher strength. There, electroluminescence light - the S2 signal - is emitted isotropically with intensity proportional to the number of ionization electrons.

In the NEXT detectors built to date, the S1 and S2 optical signals are detected by photosensor arrays with specific functions: the energy array, with low noise and singlephoton sensitivity, provides a precise measurement of the intensity of both S1 and S2, whereas the tracking array, consisting of a dense matrix of small photosensors, measures the transverse coordinates (with respect to the drift direction) of the ionization track using the S2 signal. The longitudinal coordinates are derived from the time difference between S1 and S2. The energy and tracking arrays are located, respectively, behind the cathode and anode electrodes that define the electric field. The internal walls of the detectors are covered with reflective material (e.g., PTFE) to improve light collection. We know this arrangement of the detector components as the asymmetric design (see the left panel of figure 1). Alternatively, the detector elements could be arranged symmetrically 

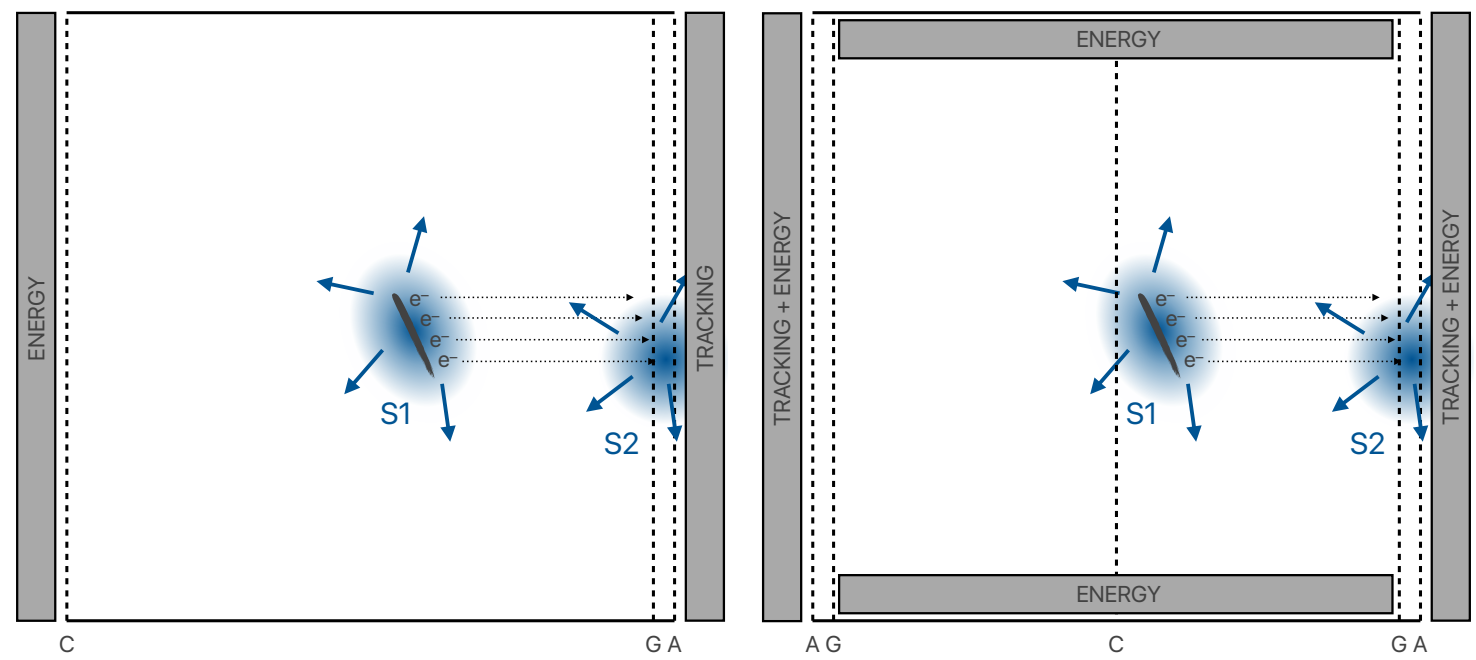

Figure 1. The NEXT detector concept in two different configurations. In the so-called asymmetric design (left panel), the active volume of the detector consists of a single drift region, between cathode $(\mathrm{C})$ and gate $(\mathrm{G})$, and a single EL gap, between gate and anode $(\mathrm{A})$. An array of photosensors behind the anode measures the start-of-event signal (S1) and the energy of the event (S2), whereas track reconstruction is performed with the $\mathrm{S} 2$ signals registered by a matrix of small photosensors placed behind the anode. The internal walls of the active volume are covered with reflective material (e.g., PTFE) to improve light collection. In the symmetric design (right panel), the active volume is divided by a central cathode into two identical drift regions equipped with an EL gap and a photosensor array that measures both tracking and energy. Alternatively, the reflective walls can be replaced by wavelength-shifting light guides coupled to photodetectors outside of the sensitive volume.

with respect to a central cathode that would divide the active volume into two identical drift regions (see the right panel of figure 1). In this symmetric design, both ends of the chamber would be equipped with an EL gap and a tracking array. These photosensors could measure as well the energy, or, as another option, the reflective walls could be replaced with photon detectors (e.g., wavelength-shifting light guides coupled to photosensors outside of the active volume). Both the asymmetric and symmetric design configurations have pros and cons. For example, for the same detector dimensions, the symmetric scheme roughly doubles the number of electronic channels, but halves the maximum drift length, easing the requirements on drift high voltage and gas purity.

Over the last decade, the NEXT Collaboration has proven the performance of the HPXeTPC technology in the key parameters required for the observation of $0 \nu \beta \beta$ decay. The NEXT concept was initially tested in small, surface-operated detectors [24-28]. This phase was followed by the underground operation at the LSC of NEXT-WhitE [29], an asymmetric, radiopure HPXeTPC containing approximately $5 \mathrm{~kg}$ of xenon at 10 bar pressure. The results obtained with NEXT-WHITE include the development of a procedure to calibrate the detector using ${ }^{83 \mathrm{~m}} \mathrm{Kr}$ decays [30], measurement of an energy resolution at $2.5 \mathrm{MeV}$ better than $1 \%$ FWHM $[31,32]$, demonstration of robust discrimination between single-electron and double-electron tracks [33], and measurement of the radiogenic background, validating the accuracy of our background model [34, 35]. 
The NEXT-100 detector [16], scheduled to start operation in 2022, constitutes the third phase of the program. It is an asymmetric HPXeTPC containing about $100 \mathrm{~kg}$ of xenon (enriched at $\sim 90 \%$ in ${ }^{136} \mathrm{Xe}$ ) at 15 bar pressure. The active region of the detector is a cylinder $130 \mathrm{~cm}$ long and $100 \mathrm{~cm}$ in diameter (about $1 \mathrm{~m}^{3}$ volume). Track reconstruction will be performed with the EL signals registered by a matrix of approximately 3600 Hamamatsu silicon photomultipliers $(\mathrm{SiPM})$ of $1.3 \times 1.3 \mathrm{~mm}^{2}$ active area placed a few millimetres beyond the anode. The event energy will be measured with an array of 60 Hamamatsu R11410-10 photomultiplier tubes (PMTs) located behind the cathode. These PMTs will also record the primary scintillation that signals the $t_{0}$ of an event. The detector inner elements are all housed inside a solid copper structure, $12 \mathrm{~cm}$ thick, contained in a stainlesssteel pressure vessel, and surrounded by a 20 -cm-thick shield made of staggered lead bricks. NEXT-100 will reach a sensitivity of about $6 \times 10^{25} \mathrm{yr}$ after a run of 3 effective years, for a predicted background rate of at most $4 \times 10^{-4}$ counts $\mathrm{keV}^{-1} \mathrm{~kg}^{-1} \mathrm{yr}^{-1}$ [17].

\section{A NEXT detector with a tonne of xenon}

The NEXT detector concept can be scaled up to $0 \nu \beta \beta$ source masses of the order of a few tonnes introducing several technological advancements that are, for the most part, already available. Figure 2 shows a possible design for a detector with an active volume of $2.6 \mathrm{~m}$ in diameter and an axial length of $2.6 \mathrm{~m}$ that would hold a mass of ${ }^{136} \mathrm{Xe}$ (when enriched to $\sim 90 \%$ in that isotope) of $1109 \mathrm{~kg}$ at 15 bar pressure. These dimensions and operational conditions are informed by R\&D performed by the Collaboration on the scalability of the NEXT-100 design in terms of number of electronic channels, size of the field-cage electrode grids and rating of the high-voltage feedthroughs. This baseline concept, referred to in what follows as NEXT-1t, includes two important design changes with respect to NEXT100: the transition to a symmetric TPC design (cf. the left and right panels of figure 1) with a central cathode and two EL gaps, and the replacement of the photodetectors in charge of the energy and $t_{0}$ measurements.

The photomultiplier tubes employed in NEXT-100 to measure energy and $t_{0}$ are one of the leading sources of radioactive background in the detector [17], and they also introduce significant mechanical complexity in the design, as they are not pressure-resistant and have to be housed in a separate low-pressure region. Silicon photomultipliers, already used in NEXT for track reconstruction, are the most obvious alternative: they are radiopure, pressure-resistant and can provide large photosensitive coverage with high granularity at acceptable cost. A dense array of SiPMs located behind the TPC anode could be in charge of both tracking and the energy measurement. However, this array would not be sensitive to the S1 signal (used to establish the $t_{0}$ ) of low-energy events, such as the

${ }^{83 \mathrm{~m}} \mathrm{Kr}$ decays used for calibration, due to the high dark-count rate (DCR) of SiPMs (at present, about $0.1 \mathrm{MHz} / \mathrm{mm}^{2}$ at $25^{\circ} \mathrm{C}$, orders of magnitude higher than that of PMTs). One possible solution involves the use of panels of double-clad wavelength-shifting (WLS) fibres covering the internal walls of the TPC. These fibres would collect the scintillation light and transport it to PMTs placed either outside the pressure vessel or in the vessel heads, behind thick copper shielding plates. Our studies indicate that this solution can achieve 

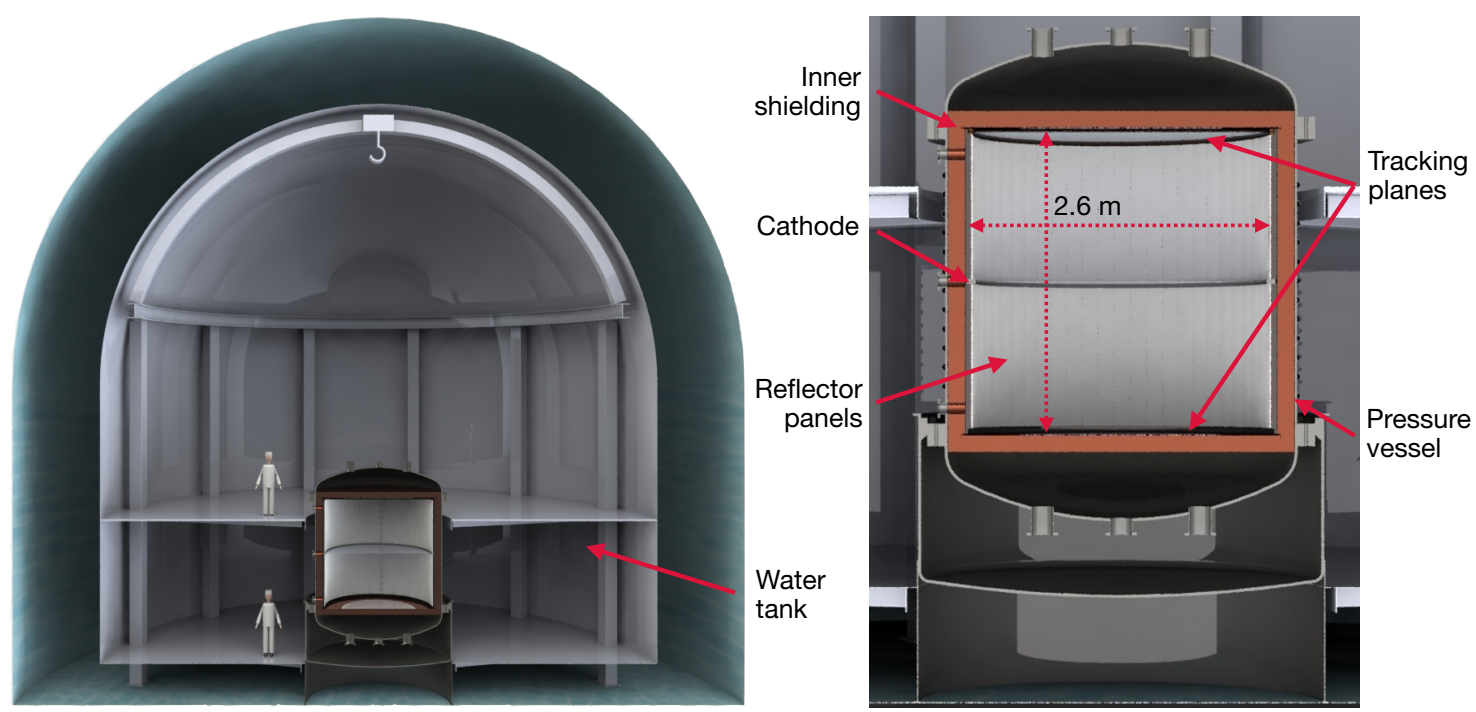

Figure 2. Left: conceptual design of a tonne-scale NEXT detector installed inside a water tank. Right: detail of the internal structures of the detector. The active volume, $2.6 \mathrm{~m}$ in diameter and height, would hold a mass of ${ }^{136}$ Xe of approximately $1109 \mathrm{~kg}$ at $15 \mathrm{bar}$.

the light collection efficiency and signal-to-noise ratio required to measure the S1 signal with similar precision to what has been achieved in NEXT-WHITE. The WLS fibres would provide as well an energy measurement complementary to that of the dense SiPM arrays. If needed, the SiPMs's DCR could be mitigated further with the use of moderately cooled gas (typically, a temperature drop of $30 \mathrm{~K}$ results in a reduction of the DCR by about an order of magnitude). The Collaboration is also exploring other readout technologies, such as high-speed image intensified cameras for tracking, or SiPMs coupled to metalenses [36].

The symmetric arrangement of the TPC halves the maximum drift length, easing the requirements on gas purity and high voltage. For example, the detector of $2.6 \mathrm{~m}$ would require $\sim 65 \mathrm{kV}$ at the central cathode to achieve a NEXT standard drift field of $500 \mathrm{~V} \mathrm{~cm}^{-1}$, a value already within the target specifications of the NEXT-100 high-voltage feedthrough. The shorter drift length would also reduce the average electron diffusion (proportional to the square root of the drift length), which impacts track reconstruction. Moreover, no buffer region would be required to protect sensors and electronics against high voltage discharges from the cathode, maximizing the isotope used for physics.

The field cage itself is expected to be an extrapolation of the current NEXT-100 design, which has been developed with scalability to the tonne scale and minimization of material mass and radioactivity as central concerns. The current design secures the field shaping rings using high-density polyethylene (HDPE) bars of the same length as the detector active region. Polytetrafluoroethylene (PTFE) panels are then attached to the HDPE bars making the light reflector seen in figure 2. These reflectors are $\sim 5 \mathrm{~mm}$ thick and constitute the majority of the mass of the field cage. If the detection of the S1 signal finally requires it, the panels of wavelength-shifting fibres would be fitted to the PTFE. The field cage is surrounded by an inner shield of $12 \mathrm{~cm}$ of copper that attenuates external 
gammas by several orders of magnitude before they reach the active volume. Developing the possibility to operate an EL readout at the meter scale has been a major R\&D effort for the Collaboration. We are testing several technologies (such as photoetching) for the production of EL meshes that can be tensioned sufficiently to operate at the field strengths envisioned and can sustain high-energy sparks without deformation.

For the purposes of this study, the detector is assumed submerged in a cylindrical water tank with dimensions to give $3 \mathrm{~m}$ of water shield on all sides of the active volume. Pure water is considered for this study, but some level of doping to improve neutron-absorption cross sections is also under consideration. If instrumented with PMTs, the tank would also allow for the tagging of muons.

In this study we consider as well the addition of either helium or a molecular gas (e.g., $\mathrm{CH}_{4}$ or $\mathrm{CF}_{4}$ ) to the xenon to reduce diffusion and improve tracking resolution [37-40].

\section{Backgrounds at the tonne scale}

Tonne-scale experiments will require significant progress in the control and understanding of backgrounds in order to achieve their physics goals. In the case of NEXT, any process capable of generating a signal-like track (see figure 3) away from the TPC walls and with energy around the $Q$ value of ${ }^{136} \mathrm{Xe}$ is a potential background source. Dominant sources are gamma rays from natural radioactivity, the decay products of ${ }^{222} \mathrm{Rn}$ and the beta decay of the long-lived neutron-capture product ${ }^{137} \mathrm{Xe}$. Other potential sources, such as the two-neutrino double-beta decay of ${ }^{136} \mathrm{Xe}$, neutrons from natural radioactivity in detector materials and surroundings, muon-spallation products or solar neutrinos, were considered and found to be subdominant. Our background model, described in detail below, has been broadly validated with the data of NEXT-WHITE [35] and will be checked again at higher precision with the NEXT-100 detector.

\subsection{Natural radioactivity in detector materials}

The main background source in NEXT is high-energy gamma radiation from long-lived radioactive contaminants present in detector materials and surroundings. Particularly troublesome are two of the gamma-ray lines emitted following the decays of ${ }^{208} \mathrm{Tl}$ and ${ }^{214} \mathrm{Bi}$, part of the thorium and uranium series, respectively. The gamma-ray line from ${ }^{208} \mathrm{Tl}\left(2614.5 \mathrm{keV}, 99.75 \%\right.$ intensity [41]) is well above $Q_{\beta \beta}=2457.8 \mathrm{keV}$, the $Q$ value of ${ }^{136} \mathrm{Xe}$, but single-electron tracks from its photopeak can lose energy via bremsstrahlung and fall in the region of interest. Likewise, gammas that interact via successive Compton scatters in close proximity may be reconstructed in some cases as a single track with energy close to $Q_{\beta \beta}$. The gamma-ray line from ${ }^{214} \mathrm{Bi}(2447.7 \mathrm{keV}, 1.55 \%$ intensity [41]) lies just below $Q_{\beta \beta}$, and thus its photopeak can overlap with the $0 \nu \beta \beta$ peak due to the finite energy resolution of the detector.

Gamma radiation emanating from laboratory walls and external support structures is unlikely to reach the inner detector through the water shielding $(3 \mathrm{~m}$ of water attenuate this gamma flux by more than 6 orders of magnitude), assuming the typical gamma flux $\left(\sim 1 \mathrm{~s}^{-1} \mathrm{~cm}^{-2}\right)$ at underground laboratories $[17,42]$. For this reason, we focus here on 

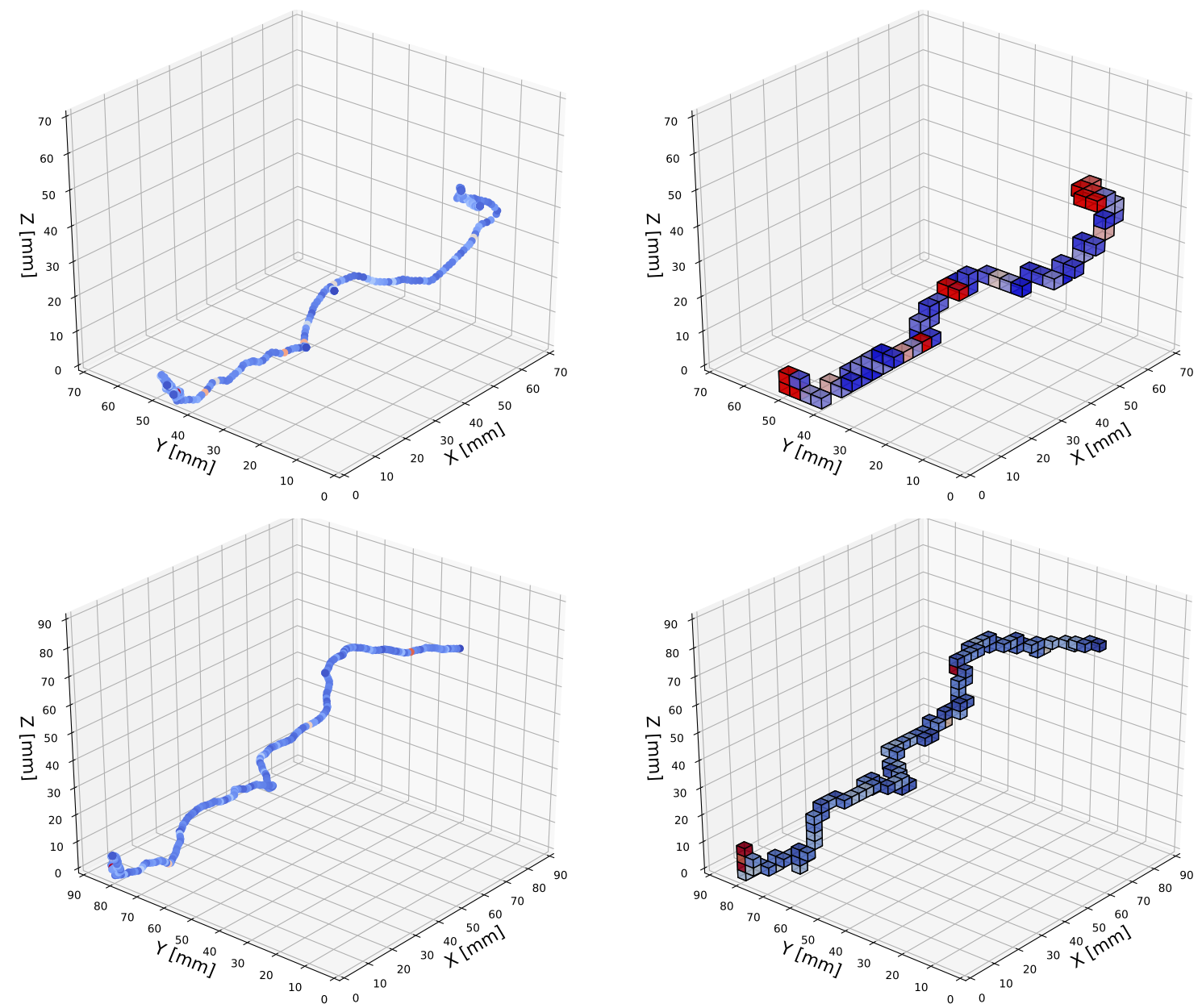

Figure 3. Monte-Carlo simulation of a $0 \nu \beta \beta$ decay of ${ }^{136} \mathrm{Xe}$ (top row) and of an electron track of the same energy (bottom row) in gaseous xenon at 15 bar. Below the so-called critical energy (about $12 \mathrm{MeV}$ in gaseous xenon), electrons lose their energy at a relatively fixed rate until they become non-relativistic; at about that time, they lose the remainder of their energy in a relatively short distance, generating a blob. Therefore, the ionization tracks left by $0 \nu \beta \beta$ events, consisting of two electrons emitted from a common vertex, feature blobs at both ends. Single-electron tracks, the main background in NEXT, only have one blob. In the left column, the tracks are shown as generated by our simulation, whereas in the right the energy deposits have been binned into $3 \times 3 \times 3 \mathrm{~mm}^{3}$ voxels to account for the effect of charge diffusion in a gas with a low-diffusion additive.

sources close to the active volume of the detector, particularly those with large mass such as the shielding copper. These backgrounds can be mitigated and understood by careful radioassay of all materials used in the construction of the detector. The NEXT Collaboration has undertaken extensive campaigns for the characterization of all materials used for the NEXT-WHITE and NEXT-100 detectors [43, 44], primarily employing gamma-ray spectroscopy with high-purity germanium detectors and inductively coupled plasma mass spectrometry (ICPMS) at the low-background facilities of LSC and Pacific Northwest National Laboratory (PNNL). For the purposes of this study, we consider, in addition to 


\begin{tabular}{|lllccc|}
\hline Material & Detector system & Method & \multicolumn{2}{c|}{ Activity $(\mu \mathrm{Bq} / \mathrm{kg})$} & Reference \\
& & & ${ }^{232} \mathrm{Th}$ & ${ }^{238} \mathrm{U}$ & \\
\hline Copper & Inner shield & ICPMS & $1.22 \pm 0.04$ & $1.28 \pm 0.09$ & This work \\
PTFE & TPC field cage & NAA & $0.103 \pm 0.012$ & $<5$ & {$[45]$} \\
Kapton & Readout planes & ICPMS & $81 \pm 15$ & $110 \pm 50$ & {$[46]$} \\
\hline
\end{tabular}

Table 1. Specific activities of ${ }^{232} \mathrm{Th}$ and ${ }^{238} \mathrm{U}$ (parents of ${ }^{208} \mathrm{Tl}$ and ${ }^{214} \mathrm{Bi}$, respectively) assumed in the background model of NEXT-1t for the most relevant materials used in the detector. Converting the activity of the parents of the natural decay chains, as measured by ICPMS and NAA, into activities of ${ }^{208} \mathrm{Tl}$ and ${ }^{214} \mathrm{Bi}$ requires the assumption of secular equilibrium.

our own measurements, others reported in the literature for similar materials with lower activity. Table 1 lists the leading contributions to the radioactivity budget of NEXT-1t. The quoted values correspond to specific activities of ${ }^{232} \mathrm{Th}$ and ${ }^{238} \mathrm{U}$, parents of the thorium and uranium decay chains. Converting those measurements into activities of ${ }^{208} \mathrm{Tl}$ and ${ }^{214} \mathrm{Bi}$, respectively, requires the assumption of secular equilibrium. The NEXT-White background prediction used this assumption for materials similar to those to be used in NEXT-100 and NEXT-1t, and the agreement between predicted and measured background rates validates it.

The material that dominates the budget is copper, given the large mass (nearly 40 tonnes) used for the inner shield. Our best activity measurement (C11000 copper supplied by Lugand Aciers, radioassayed at PNNL using ICPMS) is comparable to values reported elsewhere [47]. Further reductions in the activity of copper could be possible through electroforming [45], but this technique is slow and expensive, and thus we do not consider electroformed copper for our baseline design. However, based on the attenuation length of the ${ }^{214} \mathrm{Bi}$ and ${ }^{208} \mathrm{Tl}$ gammas in copper, manufacture of the whole mass would not be necessary to gain a significant improvement. An inner shell of $\sim 2 \mathrm{~cm}$ thickness would suffice to attenuate the flux, effectively self-shielding the copper.

After copper, PTFE and Kapton, two synthetic polymers, are the main contributors to the radioactivity budget of NEXT-1t. PTFE (about $500 \mathrm{~kg}$ ) represents a significant fraction of the mass of the TPC field cage. Here, we use activity measurements reported in the literature [45] that are significantly lower than our own for the PTFE used in NEXTWhite: $18.0(5) \mathrm{\mu Bq} / \mathrm{kg}$ of ${ }^{232} \mathrm{Th}$ and $10.4(5) \mathrm{\mu Bq} / \mathrm{kg}$ of ${ }^{238} \mathrm{U}$, measured at PNNL using ICPMS. In the case of Kapton, used as substrate for the SiPM support boards (each one is $8.4 \mathrm{~g}$ and covers $121 \mathrm{~cm}^{2}$ ), we use recently-published measurements [46].

Backgrounds from the pressure vessel as well as any additional infrastructure outside the detector are efficiently mitigated by the inner copper shielding. They are estimated to contribute at or below the $5 \%$ level to the full radioactive budget. This number is informed by experience from NEXT-WHITE and NEXT-100, where present upper limits sit at approximately $5-10 \%$ of the total activity budget $[17,35]$. Any additional external sources can be effectively mitigated by increasing the thickness of inner copper shielding without significant detriment to the total activity. 


\subsection{Radon}

Radon is another potential source of radioactive background, since it can diffuse from detector materials or the gas system and enter the active region. Only two radon isotopes, ${ }^{220} \mathrm{Rn}$ and ${ }^{222} \mathrm{Rn}$, from the thorium and uranium series, respectively, are found in significant amounts. Their production rates are similar, but the longer half-life of the latter (3.8 days versus the 55 seconds of ${ }^{220} \mathrm{Rn}$ [41]) makes it much more likely to become a background. Radon-222 undergoes two decays to produce ${ }^{214} \mathrm{Bi}$, and previous NEXT measurements show that these daughters usually plate out onto the cathode [34]. The subsequent ${ }^{214} \mathrm{Bi}$ decays on the cathode are rejected with high efficiency (through fiducial cuts) by the detection of the emitted beta electrons and coincident decays of ${ }^{214}$ Po. Rejection efficiency should only increase in a symmetric design since the cathode would be surrounded by fully instrumented volumes.

For the present study, we consider the internal radon backgrounds at a similar rate as that of the present generation of NEXT detectors [34], a conservative baseline, given the improvements in radiopurity expected in NEXT-1t. Additional contributions from airborne radon backgrounds from outside the vessel are expected to be negligible due to the surrounding water.

\subsection{Backgrounds of cosmogenic origin}

Cosmogenic backgrounds in NEXT derive from neutron capture on detector materials, especially on copper isotopes and ${ }^{136} \mathrm{Xe}$. The main source of the neutrons that induce these potential backgrounds are atmospheric muons with energies up to a few $\mathrm{TeV}$ that reach the laboratory through the rock overburden. For this study, we estimate the cosmogenic backgrounds from these muons in two example laboratory locations: one at about 3400 meters water equivalent (m.w.e.), such as Laboratori Nazionali del Gran Sasso (LNGS), and another one at 6000 m.w.e., like SNOLAB. The muon spectra are calculated using the MUSUN muon transport simulation code [48]. The most recent measurements for these locations give total fluxes of $3.432(3) \times 10^{-8} \mathrm{~cm}^{-2} \mathrm{~s}^{-1}$ for LNGS [49] and $3.31(9) \times 10^{-10} \mathrm{~cm}^{-2} \mathrm{~s}^{-1}$ for SNOLAB [50], which are used to normalize the spectra shown in figure 4.

Neutron capture produces two types of potential background: prompt activity from gamma radiation post capture, and the creation of long-lived nuclei with decays that can result in events at energies close to $Q_{\beta \beta}$. The former is dominated in NEXT by contributions from the capture of neutrons on the two main copper isotopes via the reactions ${ }^{63,65} \mathrm{Cu}(n, \gamma){ }^{64,66} \mathrm{Cu}[41]$, but there are also contributions from captures on plastics and on the steel pressure vessel. The cascade photons from all of these various reactions have energies up to tens of $\mathrm{MeV}$. As the interactions in the gas occur within a few ms of the passage of a muon, these events can be rejected without significant reduction to detector live-time introducing a veto of $2 \mathrm{~ms}$ after the tagging of a muon in the water tank or the TPC. Any remaining events contribute at a negligible level.

Non-prompt backgrounds derive from the production of long-lived isotopes that later decay with $Q$ values above $Q_{\beta \beta}$. The dominant contribution to this background comes 


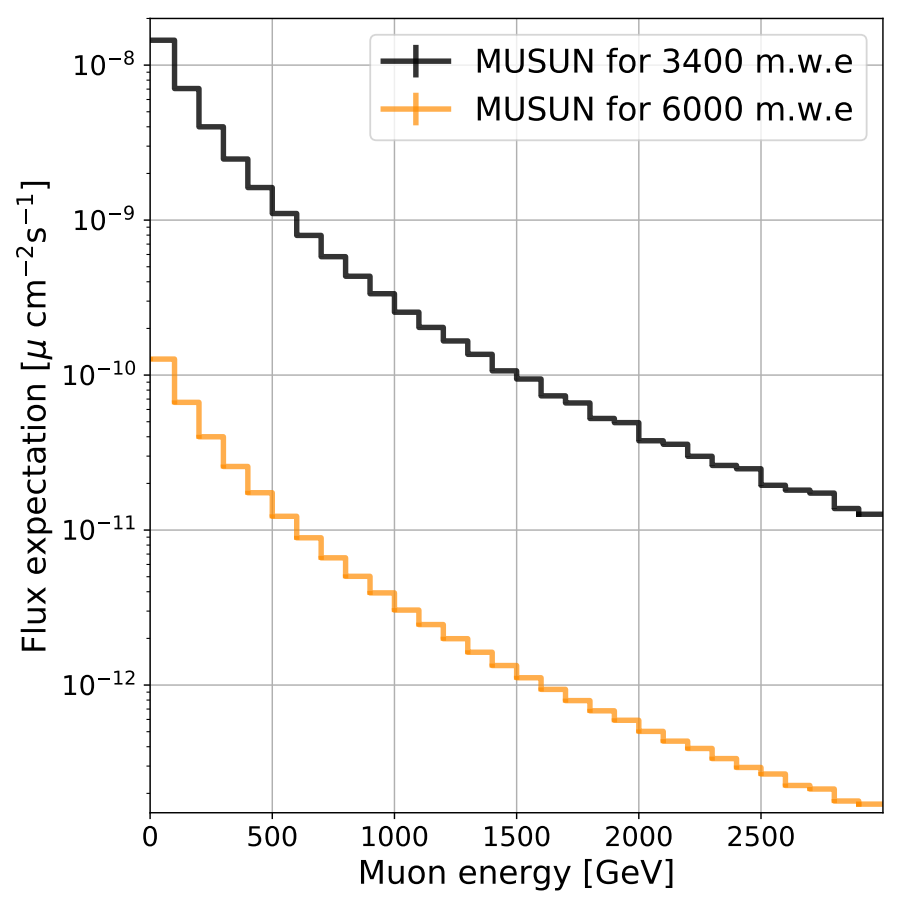

Figure 4. Expected muon flux at LNGS (3400 m.w.e.) and SNOLAB (6000 m.w.e.) estimated with the MUSUN muon-transport simulation code [48].

from the beta-emitter ${ }^{137} \mathrm{Xe}$, produced by single-neutron capture on ${ }^{136} \mathrm{Xe}$. Xenon-137 decays with a half-life of 3.8 minutes and a $Q$ value of $4.17 \mathrm{MeV}$ [41]. This background is difficult to veto by time coincidence with a detected muon due to the excessive dead-time that it would generate (for example, the anticipated muon rate at LNGS is approximately 12 muons per hour). The number of ${ }^{137} \mathrm{Xe}$ produced per muon can be directly predicted from simulation. As can be seen in figure 5, production depends nearly linearly on muon energy. Combining this prediction with the expected flux at a lab site yields the annual ${ }^{137} \mathrm{Xe}$ production rate. The integrated expectation per year for the two example labs is $131 \pm 4$ (stat.) $\mathrm{yr}^{-1}$ at LNGS and $1.40 \pm 0.04$ (stat.) $\mathrm{yr}^{-1}$ at SNOLAB, with an additional $\sim 20 \%$ systematic error expected from neutron-production model uncertainties.

\section{Signal efficiency and background rejection}

Following the same methodology used in the past for NEXT-100 [17], we can evaluate the signal efficiency and background rejection of NEXT-1t making use of large simulation datasets produced with NEXUS [51], the Geant4 [52] simulation framework developed by the Collaboration. NEXUS provides as output for each event a collection of threedimensional hits representing the ionization tracks left by charged particles in the active volume of the detector. An example of this is shown in the left column of figure 3 . The output of NEXUS is processed with a parameterization of the NEXT reconstruction that introduces detector effects such as energy and tracking resolutions, and then filtered with a cut-based event selection that we use as benchmark of the ability of a detector design 

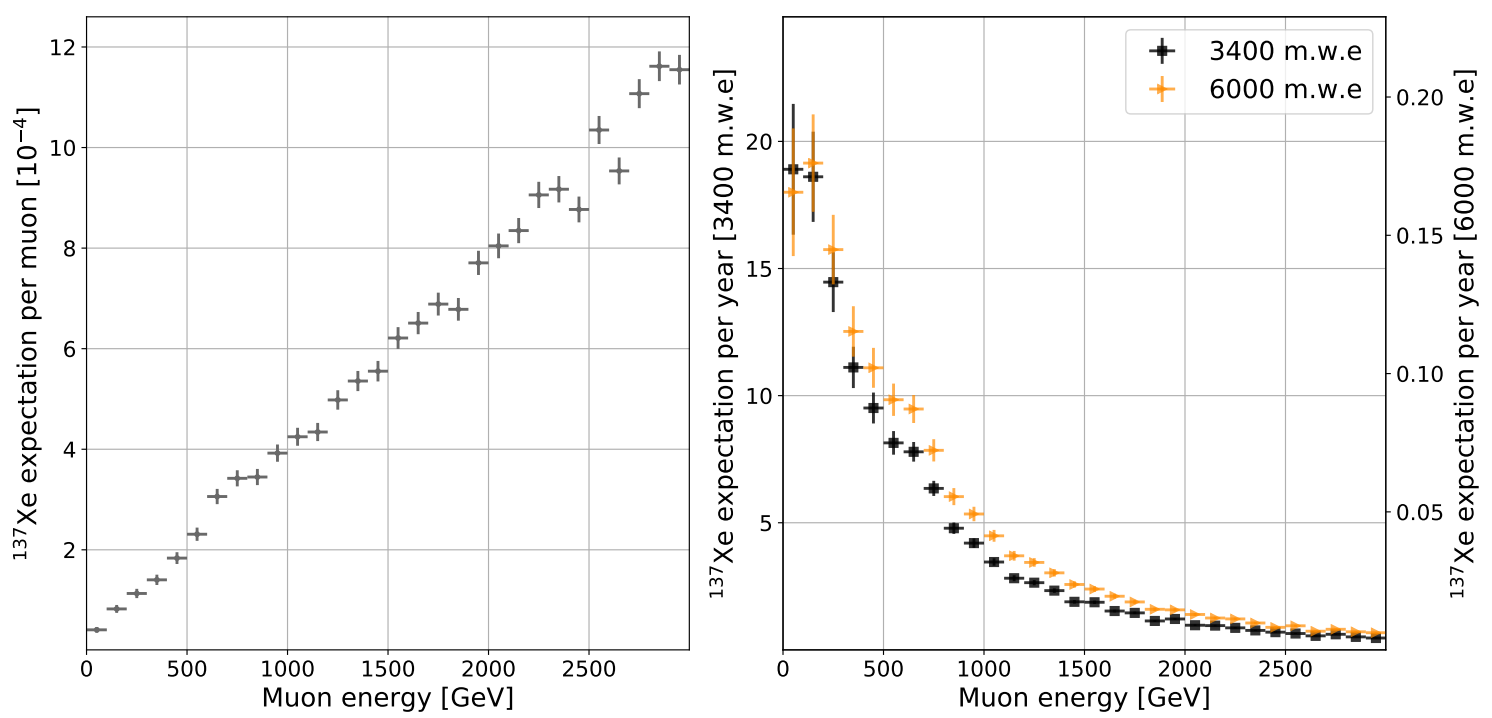

Figure 5. Expected production rate of ${ }^{137}$ Xe per interacting muon (left panel) and per year (right panel).

to reject backgrounds while retaining signal events. All samples used for this study - of the order of $1.25 \times 10^{6}$ signal events and $2 \times 10^{10}$ background events - are large enough to give a statistical error at or below $10 \%$ in the final event selection.

The initial kinematics of $0 \nu \beta \beta$ events are simulated using the DECAY0 Monte Carlo generator [53] under the assumption that $0 \nu \beta \beta$ decay is driven by the exchange of light Majorana neutrinos. NEXUS reads those events and gives them a random initial position within the xenon gas volume. Natural-radioactivity backgrounds (i.e., ${ }^{208} \mathrm{Tl}$ and ${ }^{214} \mathrm{Bi}$ ) are simulated with the radioactive-decay module in Geant4 and given initial positions uniformly distributed within the different detector volumes considered a source of background. For radon, we only consider the resulting ${ }^{214} \mathrm{Bi}$ decays coming from the cathode. For the simulation of cosmogenic backgrounds, an additional volume of a few metres of standard rock is introduced to the geometry above and around the water tank, and the muons start outside that volume. In this way, spallation neutrons produced by the interaction of cosmic-ray muons in the last few metres of the rock overburden surrounding the laboratory are included in the assessment of background.

The impact of the spatial resolution of the detector is simulated by grouping the ionization hits into cuboids (voxels) with dimensions chosen to mimic the expected diffusion conditions. For this study, we use voxels of $3 \times 3 \times 3 \mathrm{~mm}^{3}$, which we expect could be achieved through the use of gas additives to reduce diffusion. All energy deposits recorded in the detector are smeared according to a normal distribution with a standard deviation that results in an energy resolution of $0.5 \% \mathrm{FWHM}$ at $Q_{\beta \beta}$. This value approaches the intrinsic limit for xenon [24], and is expected to be achievable with the HPXeTPC technology with improvements in light collection and tracking resolution.

The left panel of figure 6 shows the energy spectra of signal and background after the application of the individual cuts of our event selection. The initial step involves 

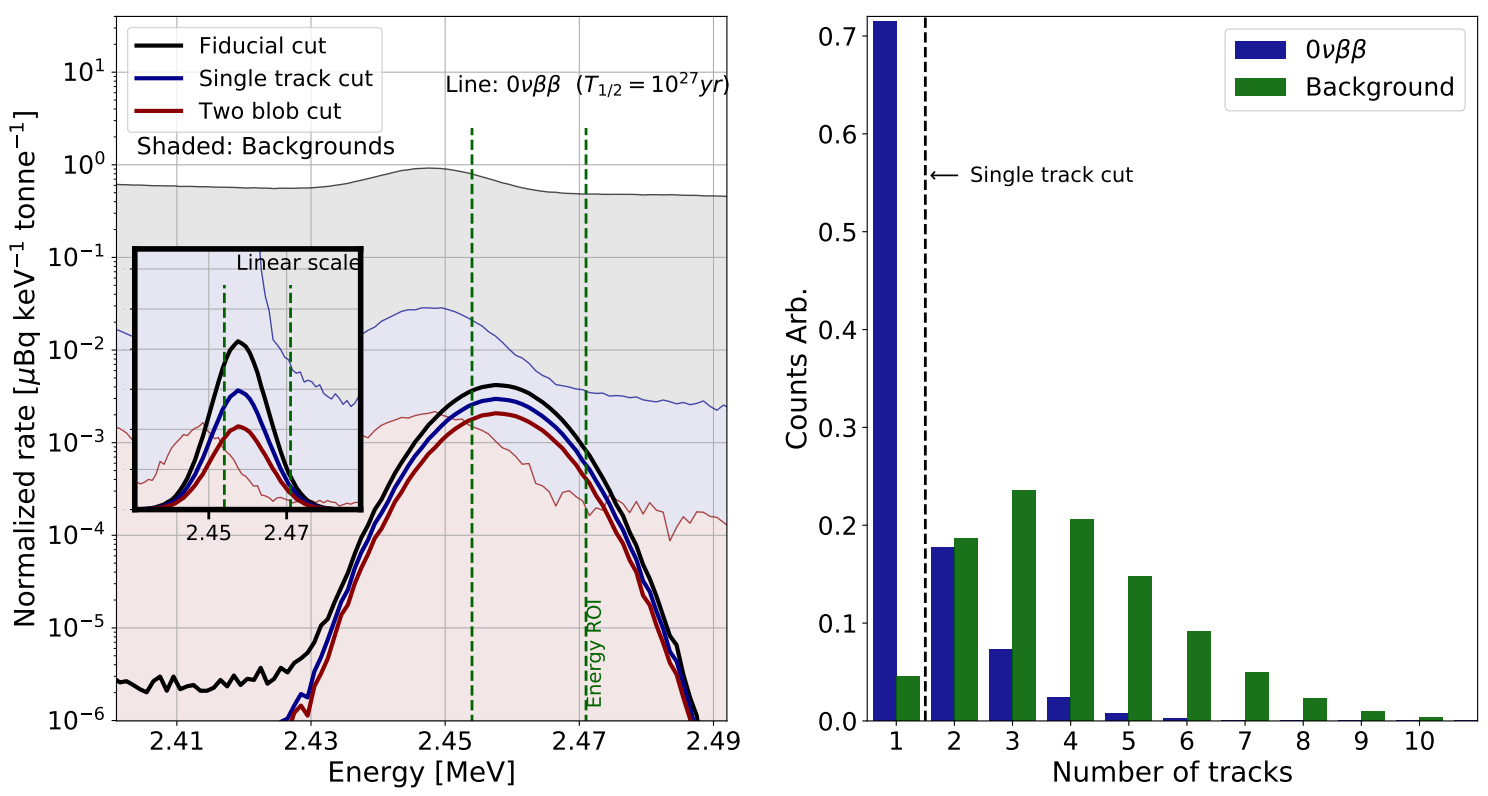

Figure 6. Left: normalized energy spectra of all simulated events after each selection cut is applied. The shaded distributions correspond to the backgrounds events, while the $0 \nu \beta \beta$ signal (whose halflife is assumed to be $10^{27} \mathrm{yr}$ for illustration purposes) is represented with solid lines. Right: track multiplicity for signal (blue bars) and background (green bars) events.

rejecting events with reconstructed energy outside the range $2.4-2.5 \mathrm{MeV}$, far from $Q_{\beta \beta}$. The surviving events are then required to have no voxels within $2 \mathrm{~cm}$ of the field cage, nor within $2 \mathrm{~cm}$ of the anode or cathode. With these first two cuts, events that obviously enter the active volume from outside or that have energies far from the region of interest are efficiently rejected.

The surviving events are subjected to the basic NEXT topological analysis: voxels are grouped into tracks according to a minimum-proximity criterion, and the resulting tracks are classified according to their topology as signal or background. The expected topology of a $0 \nu \beta \beta$-decay event (see figure 3) is a single continuous energy deposition - as the two electrons share the same initial vertex and are reconstructed together - with high-density energy deposits, referred to as blobs, at the two ends. Multi-particle background events will be reconstructed with more than one track, and those consisting of single electrons, resulting from either beta decays or the interaction of gamma radiation, while often producing a single track, can only have a blob at one extreme. The right panel in figure 6 shows the high efficiency with which the single-track requirement rejects background. There are, however, a non-negligible number of signal events reconstructed as having multiple tracks. The energy of the electrons in signal events is sufficient in many cases to produce bremsstrahlung photons that can then re-interact in the gas at a sufficient distance from the primary track to be reconstructed as separate. This mechanism affects both the track multiplicity and reconstructed energy of signal events. Recovery of these events is not considered here, but is part of wider studies. 


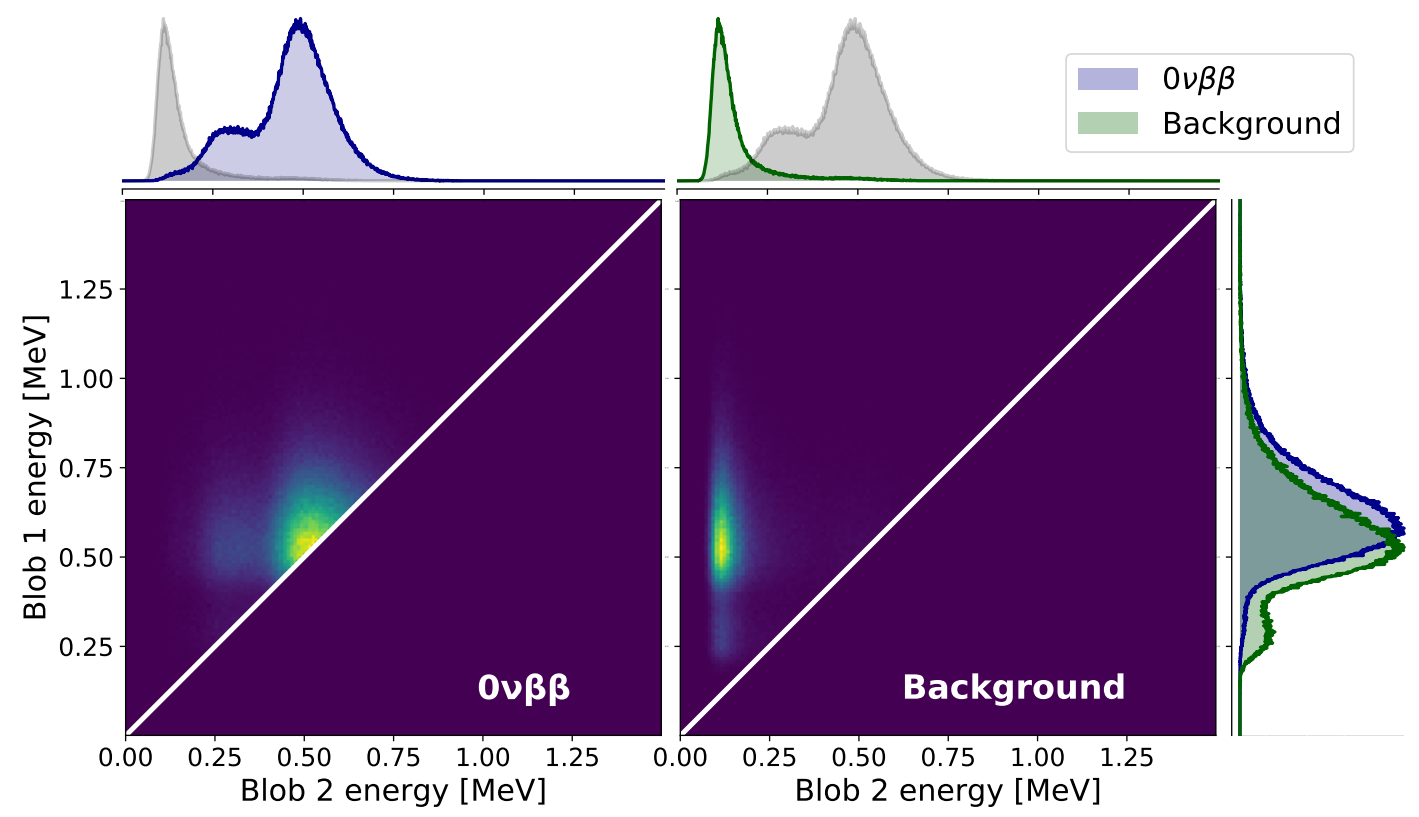

Figure 7. Blob energies of signal and background events. The blobs are defined such that blob 1 always has higher energy.

The remaining single-track events are then checked for the two-blob condition. The end-points of the tracks are identified as the two voxels at greatest distance from each other along the track. The energy in spheres of radius $15 \mathrm{~mm}$ centred at those points is integrated to give the blob energies. The blob energy is required to exceed a threshold chosen optimizing the figure of merit $\varepsilon / \sqrt{b}$, where $\varepsilon$ is the signal efficiency and $b$ is the residual background. Figure 7 shows the blob energy distributions for all signal and background events generated. The figure of merit indicates a threshold of $400 \mathrm{keV}$ as the optimal for the lower blob energy.

The subset of selected events is then reduced to those with energies falling in a region of interest (ROI) around $Q_{\beta \beta}$ (between 2454 and $2471 \mathrm{keV}$ ) that optimizes the figure of merit described above $(\varepsilon / \sqrt{b})$ for this subset of the data. The events remaining in the ROI are then used to calculate the acceptance factors: the ratio of events in the ROI to the total simulated events. A set of unique acceptance factors are calculated for each background source emanating from each detector component.

The acceptance factors are combined with the mass of each material and the expected radioactive contamination of ${ }^{208} \mathrm{Tl}$ and ${ }^{214} \mathrm{Bi}$ to yield a background index. The mass of each detector component is determined from the volume of the Geant4 geometry and the density of the material, with the exception of the readout planes, which are scaled according to surface area. The resultant activities are summarised in figure 8, where we use the term equivalent activity to describe that part of the activity that enters the selection after the application of all cuts. The acceptance factors and background index per $\mathrm{kg}$ of ${ }^{136} \mathrm{Xe}$ for the radiogenic sources considered for each detector subsystem are reported in table 2 . The contribution from cosmogenically induced ${ }^{137} \mathrm{Xe}$ is summarised in 

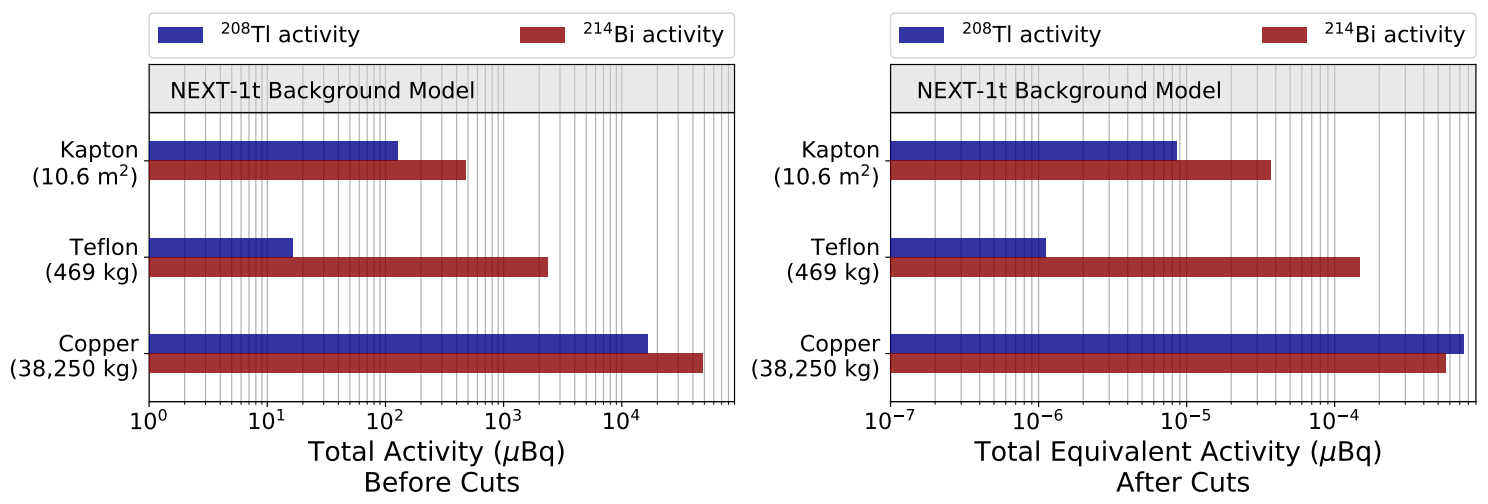

Figure 8. Left: total background activity before cuts for the dominant sources in the NEXT-1t radioactivity budget. The figures shown here result from the product of the activities listed in table 1 (assuming secular equilibrium of the decay chains) multiplied by the mass or surface of each material in NEXT-1t. In the case of Kapton, $1 \mathrm{~m}^{2}=0.413 \mathrm{~kg}$. Right: activity remaining after selection cuts.

\begin{tabular}{|lccc|}
\hline Det. system & \multicolumn{2}{c|}{ Acceptance $\left[10^{-8}\right]$} & Background index \\
& ${ }^{208} \mathrm{Tl}$ & ${ }^{214} \mathrm{Bi}$ & {$\left[\right.$ tonne $\left.^{-1} \mathrm{yr}^{-1} \mathrm{ROI}^{-1}\right]$} \\
\hline Field cage & $6.80(90)$ & $6.30(80)$ & $4.25 \times 10^{-3}$ \\
Readout planes & $6.80(90)$ & $7.80(80)$ & $1.36 \times 10^{-3}$ \\
Inner shielding & $4.50(70)$ & $1.20(70)$ & $37.23 \times 10^{-3}$ \\
Radon (cathode) & - & $0.10(10)$ & $2.72 \times 10^{-3}$ \\
\hline
\end{tabular}

Table 2. Acceptance factor (i.e., the probability of accepting a background event as signal) and resulting background indexes per unit of mass of ${ }^{136} \mathrm{Xe}$ for the natural-radioactivity background sources considered in the background model of NEXT-1t.

\begin{tabular}{|lcc|}
\hline Laboratory & Acceptance $\left[10^{-5}\right]$ & $\begin{array}{c}\text { Background index } \\
{[\text { tonne }}\end{array}$ \\
\hline LNGS & $\left.\mathrm{yr}^{-1} \mathrm{ROI}^{-1}\right]$ \\
SNOLAB & $5.68(17)$ & $6.73 \times 10^{-3}$ \\
& & $0.07 \times 10^{-3}$ \\
\hline
\end{tabular}

Table 3. Acceptance factor for the ${ }^{137}$ Xe background and resultant contribution to the background index of NEXT-1t for the two example laboratories.

table 3, where the expected number of nuclei from muon simulations is convoluted with the expected acceptance for beta electrons from the ${ }^{137}$ Xe decay. This contribution, at LNGS, is $<15 \%$ of the radiogenic background, and two orders of magnitude smaller at SNOLAB.

The effectiveness of the cut-based analysis can be seen in figure 9 , where the remaining signal and background after each cut are shown. The fiducial cut has no significant effect on the rate from ${ }^{214} \mathrm{Bi}$ and ${ }^{208} \mathrm{Tl}$ due to the relatively long interaction length of gammas at these energies. However, when these gammas interact, their energy and topology can be scrutinized and the power of the topological analysis is evident. 


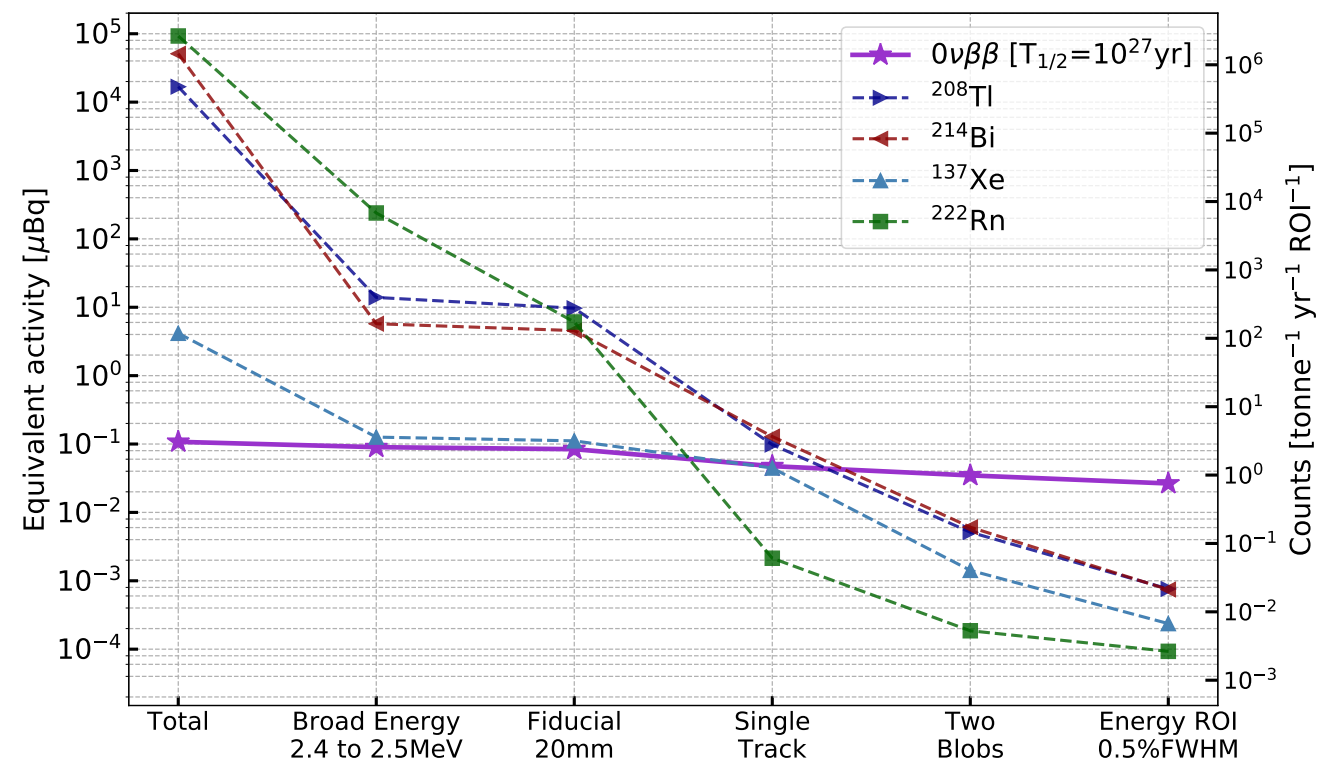

Figure 9. Equivalent background activity, background rate and signal rate as a function of the cuts for all sources considered. Here, the half life of ${ }^{136} \mathrm{Xe}$ was assumed to be $10^{27} \mathrm{yr}$, and is shown just for reference.

\section{Projected sensitivity to neutrinoless double-beta decay}

The sensitivity of an experiment searching for new phenomena is a measure of how much of the explored parameter space could be excluded by the experiment in the absence of a true signal. In our case, we define this quantity as the mean lower limit on the $0 \nu \beta \beta$-decay half-life at $90 \%$ CL that would result from many repetitions of an experiment with a null observation:

$$
\bar{T}_{1 / 2}=\log 2 \frac{N_{A}}{W} \frac{\varepsilon M t}{\bar{N}_{b}},
$$

where $N_{A}$ is the Avogadro constant, $W$ is the atomic mass of the ${ }^{136}$ Xe isotope, $\varepsilon$ is the signal detection efficiency, $M$ is the source mass, $t$ is the exposure time and $\bar{N}_{b}$ is the mean upper limit on the number of events expected under the no-signal hypothesis. Here, we use a frequentist prescription $[54,55]$ to determine $\bar{N}_{b}$ given a known, Poisson-distributed background rate. Similarly, we define the discovery potential as the $0 \nu \beta \beta$ half-life limit at 99.7\% CL resulting from our ensemble of experiments.

Table 4 lists the experimental parameters that enter the calculation of the sensitivity of NEXT-1t. The total background index is calculated by adding the radiogenic and cosmogenic contributions (see tables 2 and 3) estimated in section 5. The radiogenic background rate is increased by an additional $20 \%$ to account for subdominant sources of background, such as the pressure vessel, field-cage components or photosensors, extrapolating from our current estimates for NEXT-100 [17]. For the cosmogenic background, we use the estimation for LNGS. The resulting sensitivity can be seen in figure 10. In less than 5 years of operation, NEXT-1t could reach a half-life sensitivity of $1.4 \times 10^{27}$ yr (90\% CL), improving current limits by more than one order of magnitude. 


\begin{tabular}{|ll|}
\hline Source mass $\left({ }^{136} \mathrm{Xe}\right)$ & $1109 \mathrm{~kg}$ \\
Signal efficiency & $24.6 \%$ \\
Background rate & $0.004 \mathrm{keV}^{-1} \mathrm{t}^{-1} \mathrm{yr}^{-1}$ \\
& $0.061 \mathrm{ROI}^{-1} \mathrm{t}^{-1} \mathrm{yr}^{-1}$ \\
Energy resolution & $0.5 \% \mathrm{FWHM}^{2} 2458 \mathrm{keV}$ \\
\hline $\bar{T}_{1 / 2}(5 \mathrm{tyr})$ & $1.4 \times 10^{27} \mathrm{yr}$ at $90 \% \mathrm{CL}$ \\
$\bar{T}_{1 / 2}(10 \mathrm{tyr})$ & $2.7 \times 10^{27} \mathrm{yr}$ at $90 \% \mathrm{CL}$ \\
\hline
\end{tabular}

Table 4. Key parameters for the calculation of the sensitivity of NEXT-1t and resulting mean lower limit (at $90 \% \mathrm{CL}$ ) on the $0 \nu \beta \beta$-decay half-life for 5 and 10 tonne year of exposure.

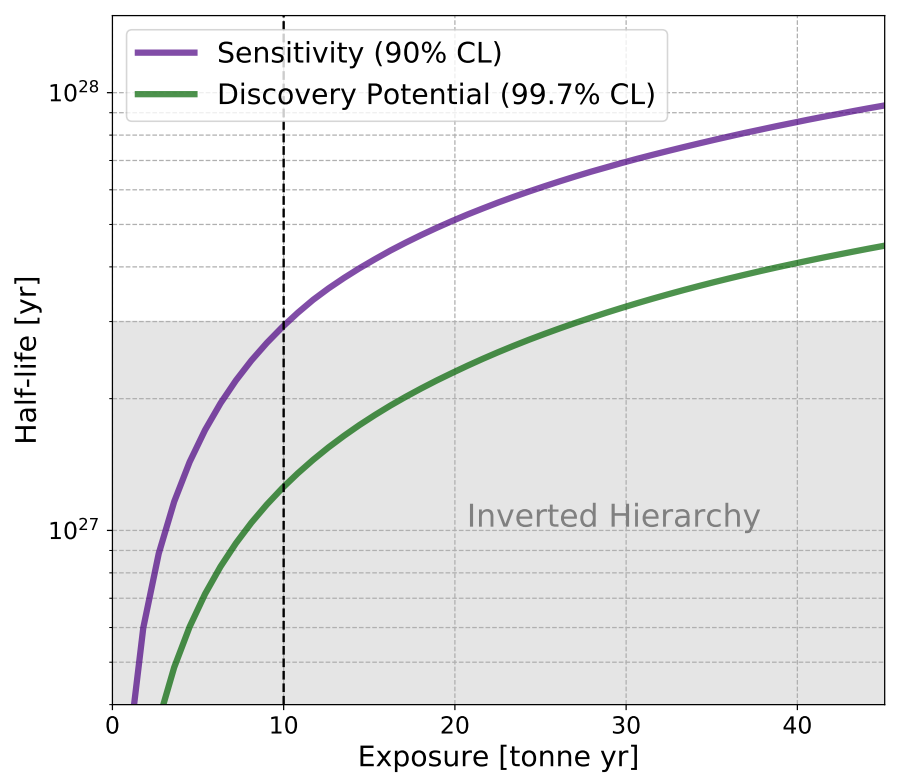

Figure 10. Projected sensitivity to the ${ }^{136} \mathrm{Xe} 0 \nu \beta \beta$ half-life and discovery potential for a NEXT tonne-scale experiment located at LNGS. The shaded region represents the inverted hierarchy under the assumption of light Majorana neutrino exchange. In order to reach exposure above $10 \mathrm{tyr}$ (indicated with a dashed line in the plot), a multi-module approach could be considered, as described in the text.

Detector performance below the levels discussed here would obviously result in a degradation of the experimental sensitivity. For example, an energy resolution of $1 \%$ FWHM at $Q_{\beta \beta}$ - worse than our best measured value [32] - translates into a reduction in sensitivity of $11 \%$, while a background rate 5 times worse causes a drop of $30 \%$. In both of these cases, the experiment would still be competitive. Improvements on these two parameters are either unlikely (an energy resolution of $0.5 \% \mathrm{FWHM}$ at $Q_{\beta \beta}$ is already near the apparent Fano limit of gaseous xenon) or pointless (a background rate an order of magnitude smaller only improves the sensitivity by $7 \%$ ). In contrast, improving the signal efficiency could be possible with more sophisticated data reconstruction and selection algorithms [56] 


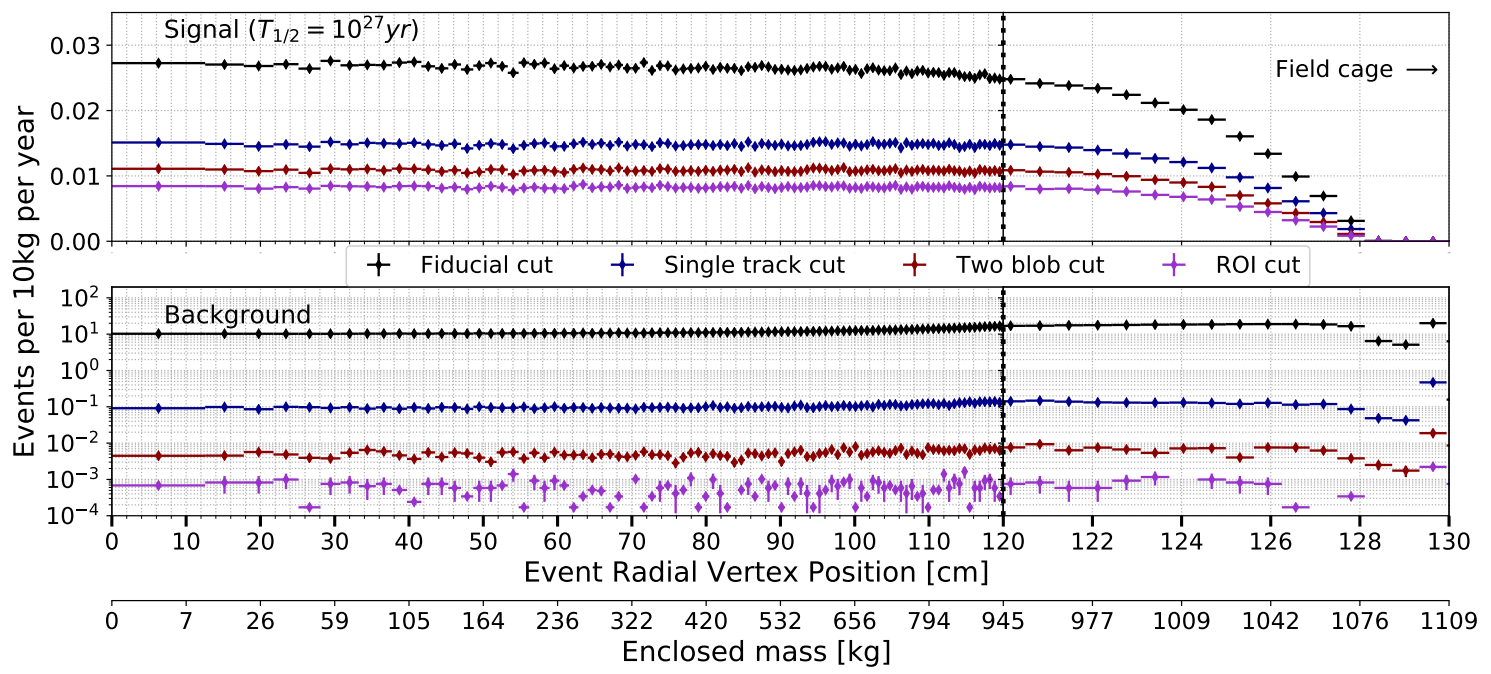

Figure 11. Event rate normalized by mass as a function of the vertex radial position in the detector for signal $\left(0 \nu \beta \beta\right.$ events) and background $\left({ }^{208} \mathrm{Tl}\right.$ and ${ }^{214} \mathrm{Bi}$ decays). In the case of signal events, the vertex corresponds to the emission point of the two electrons, while for background events it indicates the interaction point of the high-energy background gamma entering the detector. The drop in signal rate in the last $10 \mathrm{~cm}$ is an effect of considering only fully contained events and that the approximate span of $0 \nu \beta \beta$ events at the density considered is $10 \mathrm{~cm}$.

that could make use, for instance, of the signal events rejected with the single-track cut (see figure 6 ). An efficiency of $40 \%$ (compared to the $\sim 25 \%$ of our baseline scenario) would increase the sensitivity of NEXT-1t by about $60 \%$.

The baseline detector design described in this study uses $1230 \mathrm{~kg}$ of enriched xenon gas $\left(1109 \mathrm{~kg}\right.$ of $\left.{ }^{136} \mathrm{Xe}\right)$. Alternative detector masses were also studied to investigate the scaling behaviour. In addition to the default configuration, simulation sets were generated for detectors with dimensions $2 \mathrm{~m}$ in diameter and length (560 $\mathrm{kg}$ of enriched xenon) and $3 \mathrm{~m}$ in diameter and length $(1890 \mathrm{~kg})$, and no strong dependence of background index on detector size was observed. Therefore, within mechanical constraints, the detector design could be scaled up without major consequences in terms of background. This can be elaborated on further by studying the radial dependence of events, as shown in figure 11, where we represent the event rate as a function of the vertex radial position. Both signal and background events are uniformly distributed throughout the detector, since there is no self shielding like that observed in detectors using liquid xenon. This also implies that the gas phase detector utilizes a larger portion of total volume as an active detector. The radial uniformity of background events within the active volume could also allow for multiple independent detectors without the need for excess isotope, which is a major cost factor in building such experiments. In principle, the exposure from several identical tonne or multi-tonne detectors could be added to reach arbitrarily large exposures like those reached in figure 10 . 


\section{Discussion and conclusions}

Presented in this paper is an example of a tonne-scale NEXT detector capable of improving current experimental limits by more than one order of magnitude in 5 years of operation. The design of such a detector only involves incremental improvements over NEXT-100, the current stage of the NEXT project. Likewise, our sensitivity estimations are based on a well-understood background model (dominated by radiogenic sources) and event selection.

As mentioned in section 4.1, it is likely that the radiopurity expected for NEXT-100 could be significantly improved. Here we have studied the impact of the replacement of PMTs by SiPMs, the use of the purest PTFE currently available and the use of ultrapure Kapton-copper laminates for a tonne-scale experiment. However, the leading component in the NEXT-1t background model is the nearly 40 tonnes of copper that forms the inner shield.

While siting of the detector is not yet decided, it is interesting to note that NEXT-1t achieves similar sensitivity in relatively shallow laboratories to that of other detectors at much deeper sites. That being said, the possibility exists to further reduce the cosmogenic contribution by adding a small amount of ${ }^{3} \mathrm{He}$ to the gas [57]. The addition of $0.1 \%$ by mass of this isotope would reduce the number of ${ }^{137} \mathrm{Xe}$ in the active volume of the detector by an order of magnitude. In this way the NEXT design could be implemented with a lower cosmogenic background or at a shallower site without any significant impact on the performance.

High-pressure xenon gas technology offers a scalable and modular approach to $0 \nu \beta \beta$ searches, with tonne-scale sensitivity that depends primarily on exposure and not on the details of how the total active mass is deployed. Larger detectors do not exhibit an increase in background rate, and several detectors can be deployed in parallel to reach multi-tonne target masses while still taking advantage of the full volume of active isotope. The techniques presented here offer a compelling path to achieve the sensitivity needed to cross the inverted hierarchy in neutrino mass-scale sensitivity, using high-pressure xenon gas TPC experiments.

In summary, we have shown that a NEXT HPXeTPC detector holding slightly over a tonne of active isotope can efficiently reject all background sources via topological and energy analysis, achieving a background index of $\sim 0.06 \mathrm{ROI}^{-1}$ tonne $^{-1}$ year $^{-1}$ at a relatively shallow underground location (e.g. LNGS). As a baseline, we have considered a single detector with a tonne of active isotope. We envision such a detector as a stepping stone in a more ambitious program that includes the development of SMFI barium tagging for the realization of a background-free $0 \nu \beta \beta$-decay experiment that could explore half-lives up to $10^{28}$ years. 


\section{Acknowledgments}

The NEXT Collaboration acknowledges support from the following agencies and institutions: the European Research Council (ERC) under the Advanced Grant 339787-NEXT; the European Union's Framework Programme for Research and Innovation Horizon 2020 (2014-2020) under the Grant Agreements No. 674896, 690575 and 740055; the Ministerio de Economía y Competitividad and the Ministerio de Ciencia, Innovación y Universidades of Spain under grants FIS2014-53371-C04, RTI2018-095979, the Severo Ochoa Program grants SEV-2014-0398 and CEX2018-000867-S, and the María de Maeztu Program MDM2016-0692; the Generalitat Valenciana of Spain under grants PROMETEO/2016/120 and SEJI/2017/011; the Portuguese FCT under project PTDC/FIS-NUC/2525/2014 and under projects UID/FIS/04559/2020 to fund the activities of LIBPhys-UC; the Pazy Foundation (Israel) under grants 877040 and 877041; the US Department of Energy under contracts number DE-AC02-06CH11357 (Argonne National Laboratory), DE-AC0207CH11359 (Fermi National Accelerator Laboratory), DE-FG02-13ER42020 (Texas A\&M) and DE-SC0019223 / DE-SC0019054 (University of Texas at Arlington); and the University of Texas at Arlington. DGD acknowledges support from the Ramón y Cajal program (Spain) under contract number RYC-2015-18820. JM-A acknowledges support from Fundación Bancaria la Caixa (ID 100010434), grant code LCF/BQ/PI19/11690012, and from the Plan GenT program of the Generalitat Valenciana, grant code CIDEGENT/2019/049. Finally, we are grateful to the Laboratorio Subterráneo de Canfranc for hosting and supporting the NEXT experiment.

Open Access. This article is distributed under the terms of the Creative Commons Attribution License (CC-BY 4.0), which permits any use, distribution and reproduction in any medium, provided the original author(s) and source are credited.

\section{References}

[1] S. Weinberg, Baryon and Lepton Nonconserving Processes, Phys. Rev. Lett. 43 (1979) 1566 [INSPIRE].

[2] P. Minkowski, $\mu \rightarrow$ er at a Rate of One Out of $10^{9}$ Muon Decays?, Phys. Lett. B 67 (1977) 421 [INSPIRE].

[3] M. Gell-Mann, P. Ramond and R. Slansky, Complex Spinors and Unified Theories, Conf. Proc. C $\mathbf{7 9 0 9 2 7}$ (1979) 315 [arXiv:1306.4669] [InSPIRE].

[4] T. Yanagida, Horizontal gauge symmetry and masses of neutrinos, Conf. Proc. C $\mathbf{7 9 0 2 1 3 1}$ (1979) 95 [INSPIRE].

[5] R.N. Mohapatra and G. Senjanović, Neutrino Mass and Spontaneous Parity Nonconservation, Phys. Rev. Lett. 44 (1980) 912 [INSPIRE].

[6] M. Fukugita and T. Yanagida, Baryogenesis Without Grand Unification, Phys. Lett. B 174 (1986) 45 [INSPIRE].

[7] M.J. Dolinski, A.W.P. Poon and W. Rodejohann, Neutrinoless Double-Beta Decay: Status and Prospects, Ann. Rev. Nucl. Part. Sci. 69 (2019) 219 [arXiv:1902.04097] [inSPIRE]. 
[8] J. Engel and J. Menéndez, Status and Future of Nuclear Matrix Elements for Neutrinoless Double-Beta Decay: A Review, Rept. Prog. Phys. 80 (2017) 046301 [arXiv:1610.06548] [INSPIRE].

[9] S. Dell'Oro, S. Marcocci, M. Viel and F. Vissani, Neutrinoless double beta decay: 2015 review, Adv. High Energy Phys. 2016 (2016) 2162659 [arXiv:1601.07512] [INSPIRE].

[10] S.M. Bilenky and C. Giunti, Neutrinoless double-beta decay: A brief review, Mod. Phys. Lett. A 27 (2012) 1230015 [arXiv: 1203.5250] [INSPIRE].

[11] J.J. Gómez-Cadenas, J. Martín-Albo, M. Mezzetto, F. Monrabal and M. Sorel, The Search for neutrinoless double beta decay, Riv. Nuovo Cim. 35 (2012) 29 [arXiv:1109.5515] [INSPIRE].

[12] KamLAND-Zen collaboration, Search for Majorana Neutrinos near the Inverted Mass Hierarchy Region with KamLAND-Zen, Phys. Rev. Lett. 117 (2016) 082503 [Addendum ibid. 117 (2016) 109903] [arXiv: 1605. 02889] [INSPIRE].

[13] GERDA collaboration, Final Results of GERDA on the Search for Neutrinoless Double- $\beta$ Decay, Phys. Rev. Lett. 125 (2020) 252502 [arXiv: 2009.06079] [INSPIRE].

[14] A. Caldwell, M. Ettengruber, A. Merle, O. Schulz and M. Totzauer, Global Bayesian analysis of neutrino mass data, Phys. Rev. D 96 (2017) 073001 [arXiv: 1705. 01945] [InSPIRE].

[15] APPEC Committee collaboration, Double Beta Decay APPEC Committee Report, arXiv: 1910.04688 [INSPIRE].

[16] NEXT collaboration, NEXT-100 Technical Design Report (TDR): Executive Summary, 2012 JINST 7 T06001 [arXiv:1202.0721] [INSPIRE].

[17] NEXT collaboration, Sensitivity of NEXT-100 to Neutrinoless Double Beta Decay, JHEP 05 (2016) 159 [arXiv: 1511.09246] [INSPIRE].

[18] D.R. Nygren, Detecting the barium daughter in ${ }^{136}$ Xe $0-\nu \beta \beta$ decay using single-molecule fluorescence imaging techniques, J. Phys. Conf. Ser. 650 (2015) 012002 [InSPIRE].

[19] B.J.P. Jones, A.D. McDonald and D.R. Nygren, Single Molecule Fluorescence Imaging as a Technique for Barium Tagging in Neutrinoless Double Beta Decay, 2016 JINST 11 P12011 [arXiv: 1609.04019] [INSPIRE].

[20] A.D. McDonald et al., Demonstration of Single Barium Ion Sensitivity for Neutrinoless Double Beta Decay using Single Molecule Fluorescence Imaging, Phys. Rev. Lett. 120 (2018) 132504 [arXiv: 1711.04782] [INSPIRE].

[21] P. Thapa et al., Barium Chemosensors with Dry-Phase Fluorescence for Neutrinoless Double Beta Decay, Sci. Rep. 9 (2019) 15097 [arXiv: 1904.05901] [INSPIRE].

[22] I. Rivilla et al., Fluorescent bicolour sensor for low-background neutrinoless double $\beta$ decay experiments, Nature $\mathbf{5 8 3}$ (2020) 48 [INSPIRE].

[23] D. Nygren, High-pressure xenon gas electroluminescent TPC for $0 \nu \beta \beta$-decay search, Nucl. Instrum. Meth. A 603 (2009) 337 [INSPIRE].

[24] NEXT collaboration, Near-Intrinsic Energy Resolution for 30 to $662 \mathrm{keV}$ Gamma Rays in a High Pressure Xenon Electroluminescent TPC, Nucl. Instrum. Meth. A 708 (2013) 101 [arXiv: 1211.4474] [INSPIRE].

[25] NEXT collaboration, Initial results of NEXT-DEMO, a large-scale prototype of the NEXT-100 experiment, 2013 JINST 8 P04002 [arXiv:1211.4838] [INSPIRE]. 
[26] NEXT collaboration, Operation and first results of the NEXT-DEMO prototype using a silicon photomultiplier tracking array, 2013 JINST 8 P09011 [arXiv:1306.0471] [INSPIRE].

[27] NEXT collaboration, Characterisation of NEXT-DEMO using xenon $K_{\alpha} X$-rays, 2014 JINST 9 P10007 [arXiv:1407.3966] [INSPIRE].

[28] NEXT collaboration, First proof of topological signature in the high pressure xenon gas TPC with electroluminescence amplification for the NEXT experiment, JHEP 01 (2016) 104 [arXiv: 1507.05902] [INSPIRE].

[29] NEXT collaboration, The Next White (NEW) Detector, 2018 JINST 13 P12010 [arXiv: 1804.02409] [INSPIRE].

[30] NEXT collaboration, Calibration of the NEXT-White detector using ${ }^{83 m} \mathrm{Kr}$ decays, 2018 JINST 13 P10014 [arXiv: 1804.01780] [INSPIRE].

[31] NEXT collaboration, Initial results on energy resolution of the NEXT-White detector, 2018 JINST 13 P10020 [arXiv:1808.01804] [INSPIRE].

[32] NEXT collaboration, Energy calibration of the NEXT-White detector with 1\% resolution near $Q_{\beta \beta}$ of ${ }^{136} \mathrm{Xe}, J H E P 10$ (2019) 230 [arXiv:1905.13110] [InSPIRE].

[33] NEXT collaboration, Demonstration of the event identification capabilities of the NEXT-White detector, JHEP 10 (2019) 052 [arXiv: 1905.13141] [INSPIRE].

[34] NEXT collaboration, Measurement of radon-induced backgrounds in the NEXT double beta decay experiment, JHEP 10 (2018) 112 [arXiv:1804.00471] [inSPIRE].

[35] NEXT collaboration, Radiogenic Backgrounds in the NEXT Double Beta Decay Experiment, JHEP 10 (2019) 051 [arXiv:1905.13625] [INSPIRE].

[36] A.A.L. Villalpando et al., Improving the light collection efficiency of silicon photomultipliers through the use of metalenses, 2020 JINST 15 P11021 [arXiv:2007.06678] [INSPIRE].

[37] R. Felkai et al., Helium-Xenon mixtures to improve the topological signature in high pressure gas xenon TPCs, Nucl. Instrum. Meth. A 905 (2018) 82 [arXiv:1710.05600] [INSPIRE].

[38] NEXT collaboration, Electroluminescence TPCs at the Thermal Diffusion Limit, JHEP 01 (2019) 027 [arXiv: 1806.05891] [INSPIRE].

[39] NEXT collaboration, Electron Drift and Longitudinal Diffusion in High Pressure Xenon-Helium Gas Mixtures, 2019 JINST 14 P08009 [arXiv: 1902.05544] [INSPIRE].

[40] NEXT collaboration, Low-diffusion Xe-He gas mixtures for rare-event detection: Electroluminescence Yield, JHEP 04 (2020) 034 [arXiv: 1906.03984] [INSPIRE].

[41] National Nuclear Data Center, Information extracted from the NuDat 2 database (version 2.8), https://www.nndc.bnl.gov/nudat2/.

[42] M. Haffke et al., Background Measurements in the Gran Sasso Underground Laboratory, Nucl. Instrum. Meth. A 643 (2011) 36 [arXiv:1101.5298] [INSPIRE].

[43] NEXT collaboration, Radiopurity assessment of the tracking readout for the NEXT double beta decay experiment, 2015 JINST 10 P05006 [arXiv:1411.1433] [INSPIRE].

[44] NEXT collaboration, Radiopurity assessment of the energy readout for the NEXT double beta decay experiment, 2017 JINST 12 T08003 [arXiv:1706.06012] [INSPIRE].

[45] N. Abgrall et al., The Majorana Demonstrator radioassay program, Nucl. Instrum. Meth. A 828 (2016) 22 [arXiv:1601.03779] [INSPIRE]. 
[46] I.J. Arnquist, C. Beck, M.L. di Vacri, K. Harouaka and R. Saldanha, Ultra-low radioactivity Kapton and copper-Kapton laminates, Nucl. Instrum. Meth. A 959 (2020) 163573 [arXiv: 1910.04317] [INSPIRE].

[47] NEXO collaboration, nEXO Pre-Conceptual Design Report, arXiv:1805.11142 [INSPIRE].

[48] V.A. Kudryavtsev, Muon simulation codes MUSIC and MUSUN for underground physics, Comput. Phys. Commun. 180 (2009) 339 [arXiv:0810.4635] [INSPIRE].

[49] Borexino collaboration, Modulations of the Cosmic Muon Signal in Ten Years of Borexino Data, JCAP 02 (2019) 046 [arXiv: 1808. 04207] [INSPIRE].

[50] SNO collaboration, Measurement of the Cosmic Ray and Neutrino-Induced Muon Flux at the Sudbury Neutrino Observatory, Phys. Rev. D 80 (2009) 012001 [arXiv:0902.2776] [INSPIRE].

[51] J. Martín-Albo, The NEXT experiment for neutrinoless double beta decay searches, Ph.D. Thesis, Universitat de València (2015).

[52] J. Allison et al., Recent developments in Geant4, Nucl. Instrum. Meth. A 835 (2016) 186 [INSPIRE].

[53] O.A. Ponkratenko, V.I. Tretyak and Y.G. Zdesenko, The Event generator DECAY4 for simulation of double beta processes and decay of radioactive nuclei, Phys. Atom. Nucl. 63 (2000) 1282 [nucl-ex/0104018] [inSPIRE].

[54] G.J. Feldman and R.D. Cousins, A Unified approach to the classical statistical analysis of small signals, Phys. Rev. D 57 (1998) 3873 [physics/9711021] [InSPIRE].

[55] J.J. Gómez-Cadenas et al., Sense and sensitivity of double beta decay experiments, JCAP 06 (2011) 007 [arXiv: 1010.5112] [INSPIRE].

[56] NEXT collaboration, Demonstration of background rejection using deep convolutional neural networks in the NEXT experiment, JHEP 01 (2021) 189 [arXiv: 2009.10783] [INSPIRE].

[57] NEXT collaboration, Mitigation of backgrounds from cosmogenic ${ }^{137}$ Xe in xenon gas experiments using ${ }^{3}$ He neutron capture, J. Phys. G $4 \mathbf{7}$ (2020) 075001 [arXiv:2001.11147] [INSPIRE]. 